\title{
FIGO Stage II
}

National Cancer Institute

\section{Source}

National Cancer Institute. FlGO Stage II. NCI Thesaurus. Code C96252.

A FIGO stage term that applies to gynecologic cancers. For cervical cancer, it refers to cancer that invades beyond the cervix, but not to the pelvic wall or lower third of the vagina; for endometrial cancer, it refers to cancer that invades the stromal connective tissue of the cervix, but it does not extend beyond the uterus. 